Supplementary Information

\title{
Broadband Infrared Spectroscopy of Molecules in Solutions with Two Intra-pulse Difference-frequency-generated Mid-infrared Frequency Combs
}

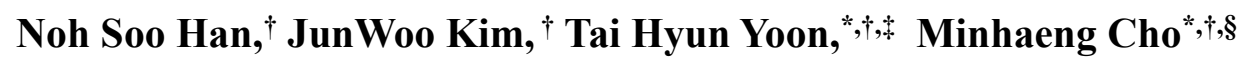 \\ ${ }^{\dagger}$ Center for Molecular Spectroscopy and Dynamics, Institute for Base Science (IBS), Seoul \\ 02841, Republic of Korea \\ ${ }^{\ddagger}$ Department of Physics, Korea University, Seoul 02841, Republic of Korea \\ ${ }^{\S}$ Department of Chemistry, Korea University, Seoul 02841, Republic of Korea \\ *E-mail: thyoon@korea.ac.kr, mcho@korea.ac.kr
}




\section{Supplementary Figures}

(a)
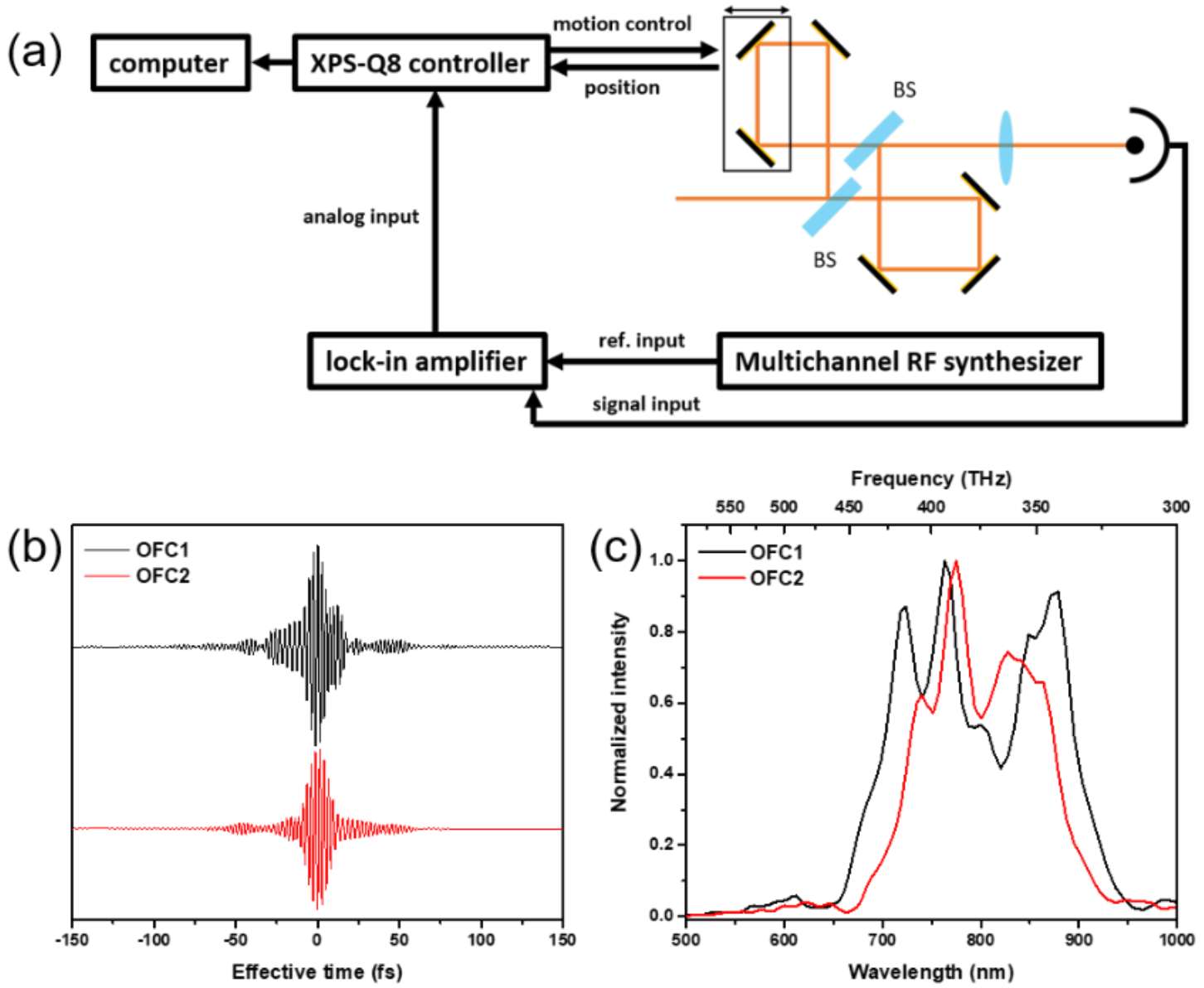

Figure S1. (a) Schematic diagram of the Mach-Zehnder interferometer setup used to measure the optical spectra of NIR pump sources and the IR spectra of mid-IR combs. The Incident beam is separated by the first beam splitter into two, and then later, they are combined by the second beam splitter. The time delay between two beams is controlled with a motorized translational stage. The recombined beam is focused on the detector. The signal is sent to a lock-in amplifier to improve the signal-to-noise ratio. The analog signal from the lock-in amplifier and the position data of the delay stage are recorded with XPS-Q8 controller, simultaneously. (b) The time-domain interferogram of OFC1 and OFC2 are shown. (c) The pulse spectra of OFC1 (black) and OFC2 (red) are shown. 
(a)

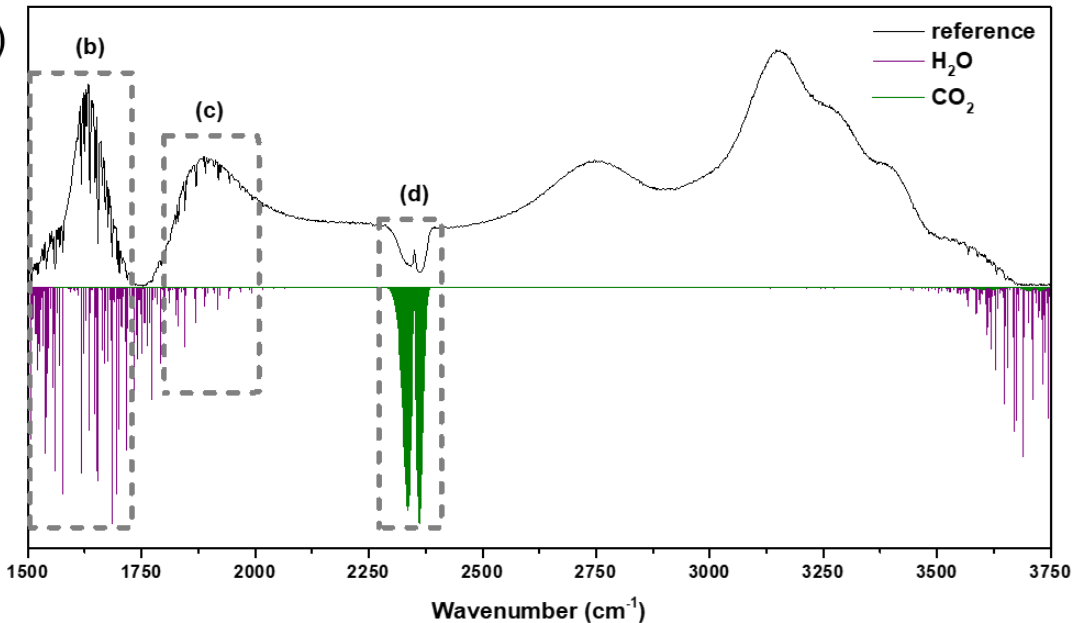

(b)
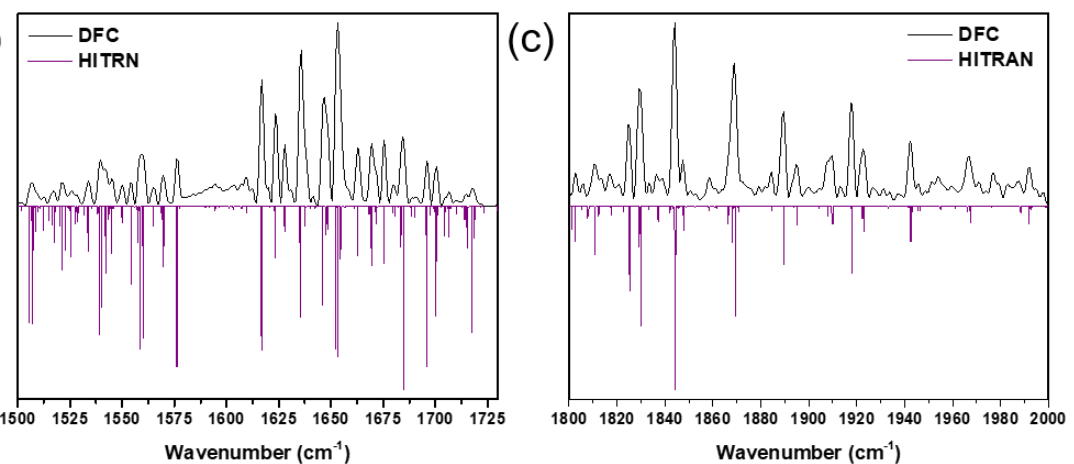

(d)

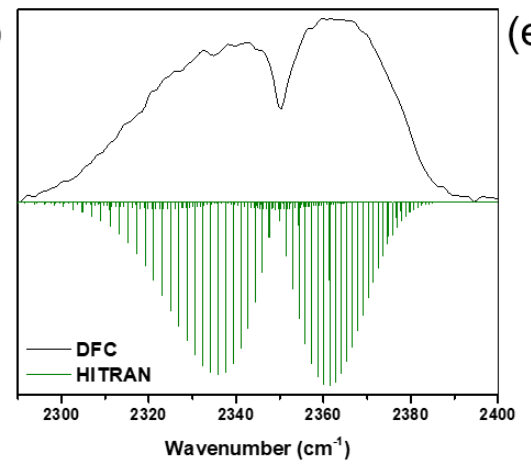

(e)

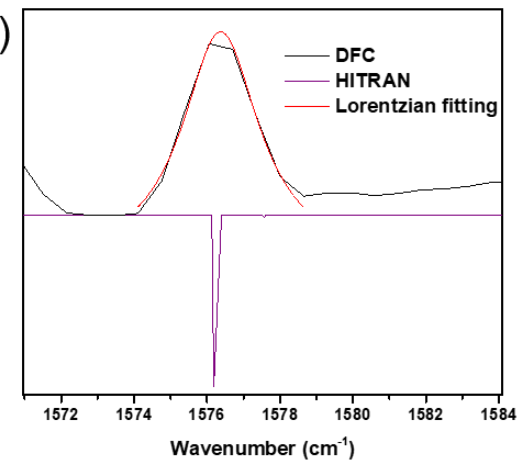

Figure S2. (a) The black line in the upper panel shows the power spectrum of mid-IR DFC data from a blank cell. (b)-(d) For the spectral band assignments and comparisons, the absorption spectra of $\mathrm{H}_{2} \mathrm{O}$ (purple) and $\mathrm{CO}_{2}$ (olive) in the gas phase that are taken from the HITRAN database are plotted here. The water bending and $\mathrm{OH}$ stretch rovibrational bands in our mid-IR DFC spectrum are in agreement with those in the HITRAN spectrum. In addition, the $P$ and $R$ branches of the asymmetric $\mathrm{CO}_{2}$ stretch rovibrational spectrum are observed in our mid-IR DFC spectrum too. One of the distinctive absorption peaks of water vapor shown in (e) is fitted with a Lorentzian function, and its full-width-at-half-maximum is found to be approximately $2 \mathrm{~cm}^{-1}$. 

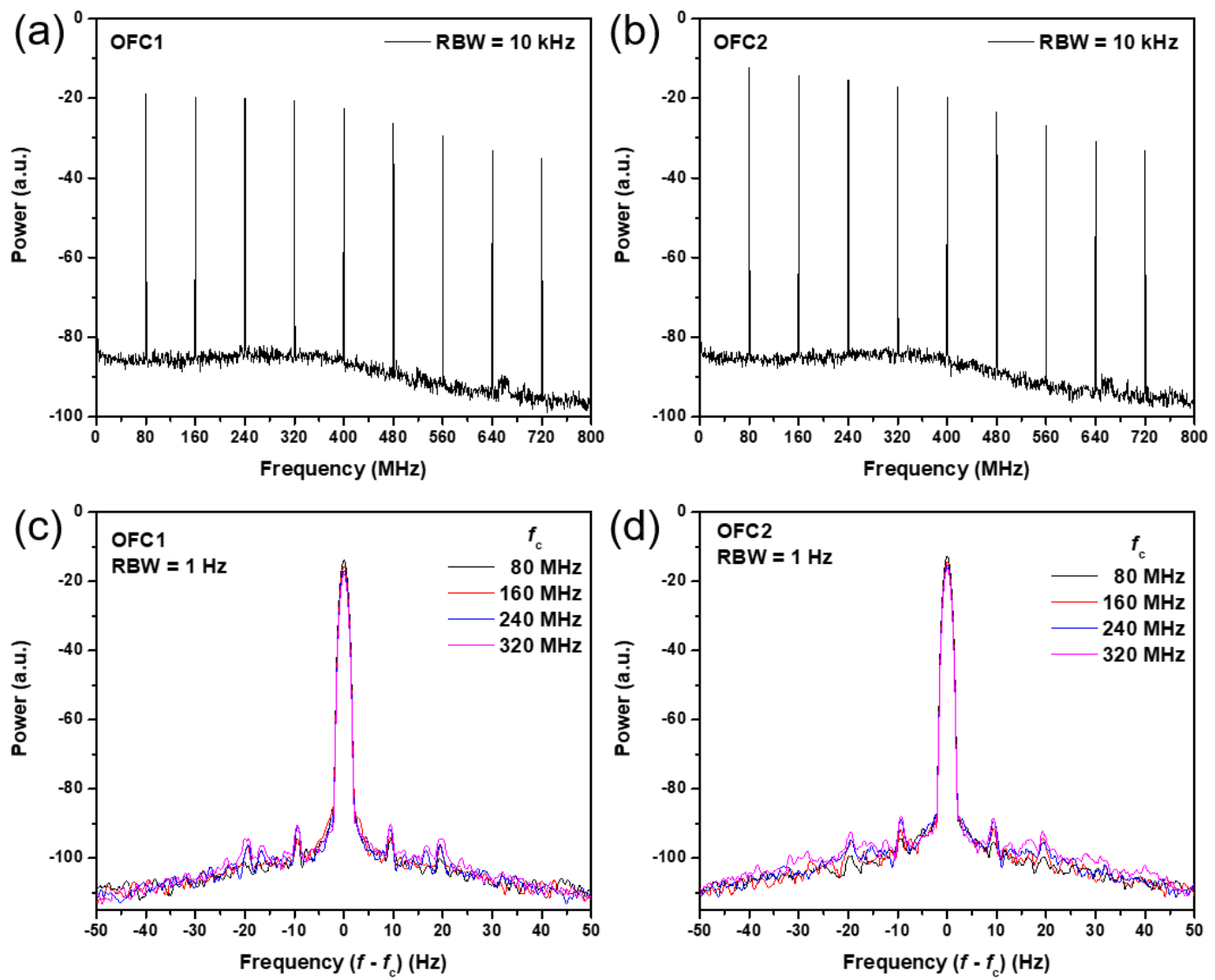

Figure S3. The RF spectra of mid-IR frequency combs from OFC1 (a) and OFC2 (b) that are measured with RF spectrum analyzer (N9000B, Keysight Technologies), where the resolution bandwidth is $10 \mathrm{kHz}$, are shown. The detectors used for the NIR OFC and mid-IR frequency combs are the biased Si detector (DET10A2, Thorlabs) and the MCT detector with a pre-amplifier (PVI-4TE-6, FIP-1k-1G-F-M4-D, VIGO), respectively. The time series of repetition rates for the NIR pump sources and the mid-IR combs were recorded with frequency counter (53230A, Keysight Technologies). The RF spectra in (a) and (b) show the frequency comb structures of the difference-frequency-generated mid-IR frequency combs. Furthermore, the mid-IR frequency combs with sub-Hz linewidth in (c) and (d) are confirmed by using the RF spectrum analyzer, whose resolution bandwidth is $1 \mathrm{~Hz}$. The linewidth of the RF beat notes of the frequency comb modes of our mid-IR comb is estimated to be sub-Hz. Due to the limited spectral resolution of our RF spectrum analyzer, we were not able to measure the spectral linewidth of the comb tooth accurately. Nevertheless, such a narrow linewidth of our mid-IR combs indicates that our mid-IR combs' repetition rates are highly stable. 

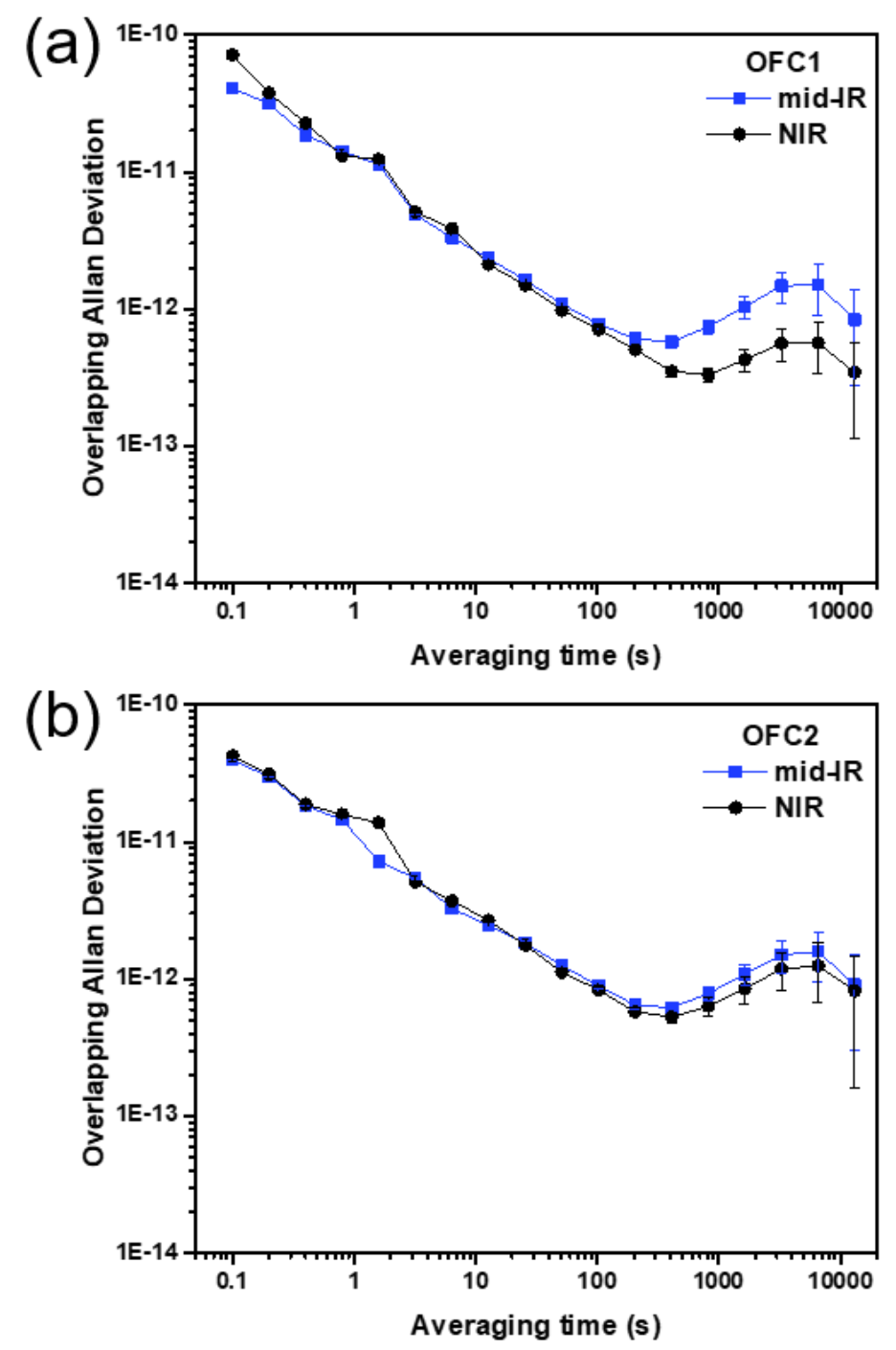

Figure S4. The time series of repetition rates of both the NIR pump sources and mid-IR combs are recorded with a frequency counter. The two figures in (a) and (b) show the overlapping Allan deviation of the NIR pump sources and the mid-IR combs from OFC1 and OFC2 over the measurement time of 20 hours. Such long-term stability originates from the active stabilization of the repetition rates of OFC1 and OFC2. Intrinsic cancellation of carrier-envelop-offset phases (CEP) during each intra-pulse DFC is an essential factor improving the stability of our mid-IR DFC spectroscopy setup. 


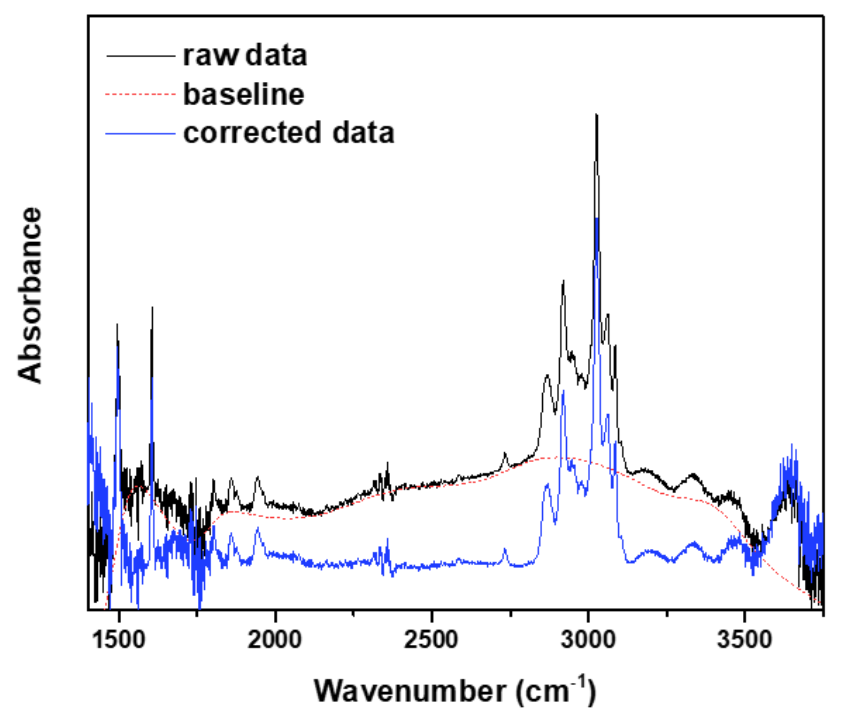

Figure S5. The raw spectrum (black) before baseline correction and the corrected spectrum (blue) after subtracting the baseline (red) from the raw spectrum are shown. The baseline is obtained by using a spline interpolation method with anchor points that can be found by examining the $2^{\text {nd }}$ derivative spectrum. 


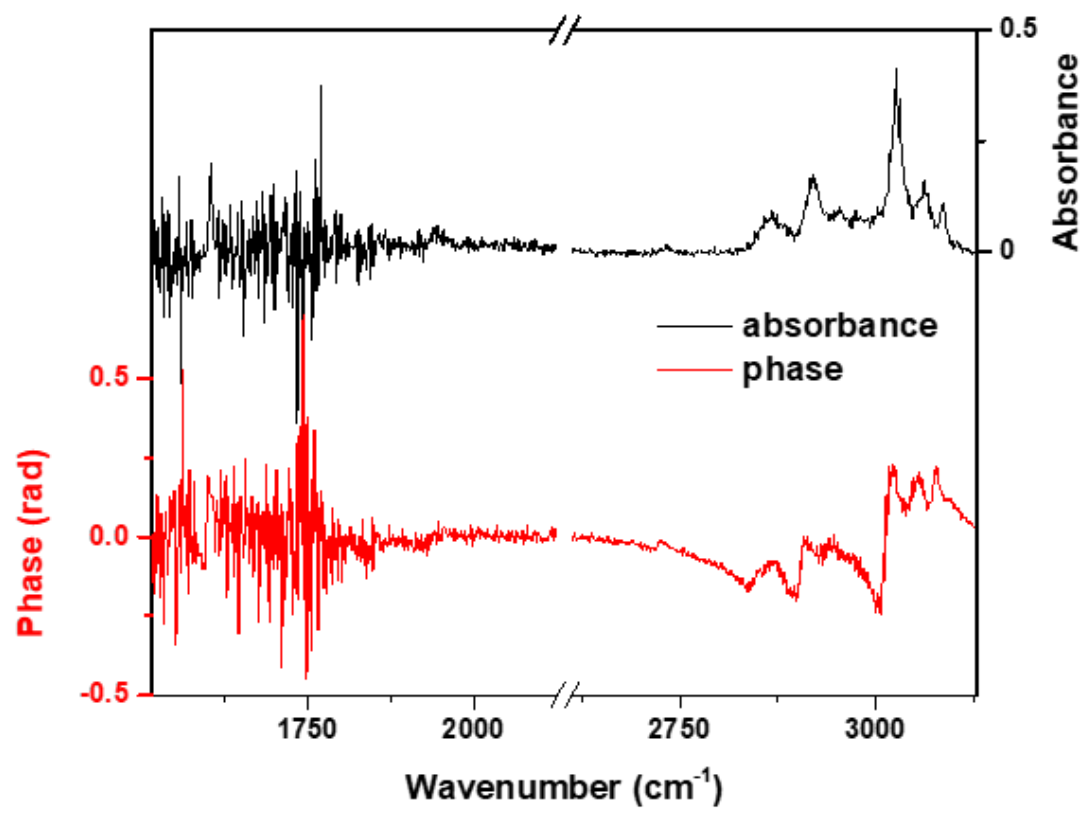

Figure S6. The absorption (black) and phase (red) spectra of liquid toluene. They are obtained by the Fourier-transform of a single interferogram of a blank (reference) cell and liquid toluene (see Fig.4(a)). 